\title{
Some Embedding Theorems on the Nikolskii-Morrey Type Spaces
}

\author{
Ali Akbulut ${ }^{1}$, Ahmet Eroglu ${ }^{2}$ and Alik M. Najafov ${ }^{34^{*}}$ \\ ${ }^{1}$ Department of Mathematics, Ahi Evran University, Kirsehir, Turkey \\ ${ }^{2}$ Nigde University, Department of Mathematics, Nigde, Turkey \\ ${ }^{3}$ Institute of Mathematics and Mechanics of NAS of Azerbaijan, Baku, Azerbaijan \\ ${ }^{4}$ Azerbaijan University of Architecture and Construction, Baku, Azerbaijan \\ Email: nadjafov@rambler.ru
}

\begin{abstract}
In the paper the Nikolskii-Morrey type spaces $H_{p, \varphi, \beta}^{l}(G)$ were introduced and studied. Some embedding theorems are obtained in $H_{p, \varphi, \beta}^{l}(G)$ with the help of Nikolskii type integral representation.
\end{abstract}

Keywords: Nikolskii-Morrey type spaces, integral representation, embedding theorems, generalized Holder condition

\section{Introduction}

In the paper, we introduce a Nikolskii-Morrey type space with parameters. By $H_{p, \varphi, \beta}^{l}(G)$ we denote the spaces of all functions $f \in L_{1}^{\text {loc }}(G)\left(m_{i}>l_{i}-k_{i}>0, i=1,2, \ldots, n\right)$ with the finite norm

$$
\begin{aligned}
& \|f\|_{H_{p, \varphi, \beta}^{l}(G)}=\|f\|_{p, \varphi, \beta ; G} \\
& +\sum_{i=1}^{n} \sup _{0<h<h_{0}} \frac{\left\|\Delta_{i}^{m_{i}}\left(\varphi_{i}(h), G_{\varphi(h)}\right) D_{i}^{k_{i}} f\right\|_{p, \varphi, \beta}}{\varphi_{i}(h)^{l_{i}-k_{i}}},
\end{aligned}
$$

where

$$
\|f\|_{p, \varphi, \beta ; G}=\|f\|_{L_{p, \varphi, \beta}(G)}=\sup _{x \in G, t>0}\left(\left|\varphi\left([t]_{1}\right)\right|^{-\beta}\|f\|_{p, G_{\varphi(t)}(x)}\right),
$$

$l \in(0, \infty)^{n}, m_{i} \in \mathbb{N}, k_{i} \in \mathbb{N}_{0}, p \in[1, \infty),[t]_{1}=\min \{1, t\}, \varphi(t)=\left(\varphi_{1}(t), \ldots, \varphi_{n}(t)\right),\left|\varphi\left([t]_{1}\right)\right|^{-\beta}=$ $\prod_{j=1}^{n}\left(\varphi_{j}\left([t]_{1}\right)\right)^{-\beta_{j}}, \beta_{j} \in[0,1], j=1,2, \ldots, n$.

Denote by $\mathbb{A}$ the set of vector functions $\varphi(t)=\left(\varphi_{1}(t), \ldots, \varphi_{n}(t)\right)$ with Lebesgue measurable functions $\varphi_{j}(t)>0, t>0, \lim _{t \rightarrow+0} \varphi_{j}(t)=0, \lim _{t \rightarrow+\infty} \varphi_{j}(t)=\infty, j=1,2, \ldots, n$.

For any $x \in R^{n}$,

$$
G_{\varphi(t)}(x)=G \cap I_{\varphi(t)}(x)=G \cap\left\{y:\left|y_{j}-x_{j}\right|<\frac{1}{2} \varphi_{j}(t), \quad j=1,2, \ldots, n\right\} .
$$

Let for any $t>0,\left|\varphi\left([t]_{1}\right)\right| \leq C$, where $C$ is positive constant. Then the embeddings $L_{p, \varphi, \beta}(G) \rightarrow L_{p}(G)$ and $H_{p, \varphi, \beta}^{l}(G) \rightarrow H_{p}^{l}(G)$ hold, i.e

$$
\begin{aligned}
\|f\|_{p, G} & \leq c\|f\|_{p, \varphi, \beta ; G}, \\
\|f\|_{H_{p}^{l}(G)} & \leq c\|f\|_{H_{p, \varphi, \beta}^{l}(G)} .
\end{aligned}
$$

Note that the spaces $L_{p, \varphi, \beta}(G)$ and $H_{p, \varphi, \beta}^{l}(G)$ are Banach spaces. The completeness of these spaces automatically implies from completeness of $L_{p}$ and $H_{p}^{l}$. The space $H_{p, \varphi, \beta}^{l}(G)$, when $\varphi_{j}(t)=t^{\chi_{j}}, \beta_{j}=\frac{a_{j}}{p}$ $(j=1, \ldots, n)$ coincides with the space $H_{p, a, \chi}^{l}(G) \equiv H_{p, \lambda}^{l}$ introduced by J.Ross [13], in the case $\beta_{j}=$ $0(j=1, \ldots, n)$ it coincides with the Nikolski space $H_{p}^{l}(G)$. The space $W_{p, \varphi, \beta}^{l}(G)$ was introduced and studied in [12]. The spaces of such type with different norms were introduced and studied in [2]-[11].

Note some properties of the spaces $L_{p, \varphi, \beta}(G)$.

1. The space $L_{p, \varphi, \beta}(G)$ is complete. 
Proof. Let $\left\{f_{i}\right\}_{i=1}^{\infty}$ be the fundamental sequences in $L_{p, \varphi, \beta}(G)$, i.e. for any $i, j \rightarrow \infty$

$$
\left\|f_{i}-f_{j}\right\|_{L_{p, \varphi, \beta}(G)} \rightarrow 0,
$$

It means that $\forall \varepsilon>0, \exists n_{0} \in N, \forall i, j>n_{0}$

$$
\left\|f_{i}-f_{j}\right\|_{L_{p, \varphi, \beta}(G)}<\varepsilon
$$

In other words $\forall \varepsilon>0, \exists n_{0} \in N, \forall i, j>n_{0}$

$$
\sup _{x \in G, t>0}\left(\left|\varphi\left([t]_{1}\right)\right|^{-\beta}\left\|f_{i}-f_{j}\right\|_{p, G_{\varphi(t)}(x)}\right)<\varepsilon
$$

and for any $x \in G, \forall t>0$

$$
\left\|f_{i}-f_{j}\right\|_{p, G_{\varphi(t)}(x)}<\varepsilon
$$

i.e. $\left\{f_{i}\right\}_{i=1}^{\infty}$ is a Cauchy sequence in $L_{p}\left(G_{\varphi(t)}(x)\right)$. The space $L_{p}(G)$ is complete, therefore there is a function $f_{0} \in L_{p}(G), i \rightarrow \infty$, for $x \in G$, for any $t>0, \forall \varepsilon>0$

$$
\left\|f_{i}-f_{0}\right\|_{L_{p}\left(G_{\varphi(t)}(x)\right)} \rightarrow \varepsilon
$$

then

$$
\left|\varphi\left([t]_{1}\right)\right|^{-\beta}\left\|f_{i}-f_{0}\right\|_{L_{p}\left(G_{\varphi(t)}(x)\right)} \rightarrow \varepsilon
$$

i.e.

$$
\begin{gathered}
\left\|f_{i}-f_{0}\right\|_{p, \varphi, \beta ; G}<\varepsilon, \\
\left\|f_{0}\right\|_{p, \varphi, \beta ; G}=\left\|f_{i}-f_{0}\right\|_{L_{p, \varphi, \beta}(G)}+\left\|f_{i}\right\|_{L_{p, \varphi, \beta}(G)}<\varepsilon_{1}+M=\varepsilon_{2}
\end{gathered}
$$

$\left\|f_{0}\right\|_{p, \varphi, \beta ; G}<\varepsilon_{2}, f_{0} \in L_{p, \varphi, \beta}(G)$.

2. Let $G$ be a bounded domain and $p \leq q ; \varphi(t) \leq \psi(t)(t>0) ; \exists c>0, \forall t \in(0,1),|\psi(t)|^{\beta_{1}} \leq c|\varphi(t)|^{\beta}$, and then $L_{q, \psi, \beta_{1}}(G) \rightarrow L_{p, \varphi, \beta}(G)$ and there exists $C>0$ such that

$$
\|f\|_{p, \varphi, \beta ; G} \leq C\|f\|_{q, \psi, \beta_{1} ; G} .
$$

Proof. . For any $t>0, x \in G$ we have

$$
\begin{gathered}
\left|\varphi\left([t]_{1}\right)\right|^{-\beta}\|f\|_{p, G_{\varphi(t)}(x)} \\
\leq\left|\varphi\left([t]_{1}\right)\right|^{-\beta}\left(m e s G_{\varphi(t)}(x)\right)^{\frac{1}{p}-\frac{1}{q}}\left|\psi\left([t]_{1}\right)\right|^{\beta_{1}}\left|\psi\left([t]_{1}\right)\right|^{-\beta_{1}}\|f\|_{q, G_{\psi(t)}(x)}
\end{gathered}
$$

and

$$
\|f\|_{p, \varphi, \beta ; G} \leq C\|f\|_{q, \psi, \beta_{1} ; G} .
$$

Definition 1.1. The open set $G \subset R^{n}$ is said to be an open set with condition of flexible $\varphi$-horn if for some $\theta \in(0,1]^{n}, T \in(0, \infty)$ for any $x \in G$ there exists the vector-function

$$
\rho(\varphi(t), x)=\left(\rho_{1}\left(\varphi_{1}(t), x\right), \ldots, \rho_{n}\left(\varphi_{n}(t), x\right)\right), \quad 0 \leq t \leq T
$$

with the following properties:

1) For all $j=1,2, \ldots, n, \rho_{j}\left(\varphi_{j}(t), x\right)$ are absolutely continuous on

$[0, T],\left|\rho_{j}\left(\varphi_{j}(t), x\right)\right| \leq 1$ for almost all $t \in[0, T]$,

2) $\rho_{j}(0, x)=0, x+V(x, \theta)=x+\bigcup_{0 \leq t \leq T}[\rho(\varphi(t), x)+\varphi(t) \theta I] \subset G$.

In particular, for $\varphi(t)=t^{\lambda},\left(t^{\lambda}=\left(t^{\lambda_{1}}, t^{\lambda_{2}}, \ldots, t^{\lambda_{n}}\right)\right)$ and $\theta_{j}=\theta^{\lambda_{j}}(j=1, \ldots, n)$ the set $x+V(x, \theta)$ is called the flexible $\lambda$-horn introduced in [1]. 
Assuming that $\varphi_{j}(t)(j=1,2, \ldots, n)$ are also differentiable on $[0, T]$, we can show that for $f \in H_{p}^{l}(G)$ determined in $n$ - dimensional domains, satisfying the condition of flexible $\varphi$-horn, it holds the following integral representation $(\forall x \in U \subset G)$

$$
\begin{aligned}
D^{\nu} f(x) & =\bar{f}_{\varphi(t)}^{(\nu)}(x)+(-1)^{|\nu|} \sum_{i=1}^{n} \int_{0}^{T} \int_{R^{n}} \int_{-\infty}^{+\infty} K_{i}^{(\nu)}\left(\frac{y}{\varphi(t)}, \frac{\rho(\varphi(t, x))}{\varphi(t)}\right) \\
& \times \zeta_{i}\left(\frac{u}{\varphi_{i}(t)}, \frac{\rho_{i}\left(\varphi_{i}(t, x)\right)}{\varphi_{i}(t)}, \frac{1}{2} \rho_{i}^{\prime}(\varphi(t), x)\right) \Delta_{i}^{m_{i}}\left(\varphi_{i}(\delta) u\right) \\
\times & \\
\bar{f}_{\varphi(t)}^{(\nu)}(x) & =\prod_{j=1}^{n} \varphi_{j}^{-2-\nu_{j}}(t) \int_{j=1} \int_{R^{n}} \Omega^{(\nu)}\left(\frac{y}{\varphi(T)}, \frac{\rho(\varphi(t), x)}{2 \varphi(t)}\right) \\
\times & \Omega\left(\frac{z}{\varphi(T)}, \frac{\rho(\varphi(t), x)}{2 \varphi(t)}\right) f(x+y+z) d y d z .
\end{aligned}
$$

Let $M_{i}(\cdot, y) \in C_{0}^{\infty}\left(R^{n}\right)$ be such that

$$
S\left(M_{i}\right) \subset I_{\varphi(t)}=\left\{y:\left|y_{j}\right|<\frac{1}{2} \varphi_{j}(t), \quad j=1,2, \ldots, n\right\} .
$$

Assume $0<T \leq 1$ is fixed and

$$
V=\bigcup_{0<t \leq T}\left\{y: \frac{y}{\varphi(t)} \in S\left(M_{i}\right)\right\} .
$$

It is clear that $V \subset I_{\varphi(t)}$. Let $U+V \subset G$.

Lemma 1.2. Let $1 \leq p \leq q \leq r \leq \infty ; 0<\eta, t<T \leq 1, \nu=\left(\nu_{1}, \nu_{2}, \ldots, \nu_{n}\right), \nu_{j} \geq 0$ are integers, $j=1,2, \ldots, n ; \Delta_{i}^{m_{i}}(h) \in L_{p, \varphi, \beta}(G)$ and let

$$
\begin{gathered}
Q_{T}^{i}=\int_{0}^{T} \prod_{j=1}^{n}\left(\varphi_{j}(t)\right)^{-\nu_{j}-\left(1-\beta_{j} p\right)\left(\frac{1}{p}-\frac{1}{q}\right)} \frac{\varphi_{i}^{\prime}(t)}{\left(\varphi_{i}(t)\right)^{1-l_{i}}} d t<\infty, \\
A(x)=\prod_{j=1}^{n} \int_{R^{n}} \int_{R^{n}} f(x+y+z) \Omega^{\nu}\left(\frac{y}{\varphi(t)}, \frac{\rho(\varphi(t), x)}{2 \varphi(t)}\right) \\
\times \Omega\left(\frac{z}{\varphi(T)}, \frac{\rho(\varphi(t), x)}{2 \varphi(t)}\right) f(x+y+z) d y d z \\
H_{\eta}^{i}(x)=\int_{0}^{\eta} L_{i}(x, t) \prod_{j=1}^{n}\left(\varphi_{j}(t)\right)^{\nu_{j}-2} \frac{\varphi_{i}^{\prime}(t)}{\varphi_{i}(t)} d t \\
H_{\eta T}^{i}(x)=\int_{\eta}^{T} L_{i}(x, t) \prod_{j=1}^{n}\left(\varphi_{j}(t)\right)^{\nu_{j}-2} \frac{\varphi_{i}^{\prime}(t)}{\varphi_{i}(t)} d t
\end{gathered}
$$

where

$$
\begin{aligned}
L_{i}(x, t) & =\int_{R^{n}} \int_{-\infty}^{+\infty} M_{i}\left(\frac{y}{\varphi(t)}, \frac{\rho(\varphi(t), x)}{\varphi(t)}\right) \\
& \times \zeta_{i}\left(\frac{u}{\varphi_{i}(t)}, \frac{\rho_{i}\left(\varphi_{i}(t), x\right)}{2 \varphi_{i}(t)}, \frac{1}{2} \rho_{i}^{\prime}\left(\varphi_{i}(t), x\right)\right) \Delta_{i}^{m_{i}}\left(\varphi_{i}(\delta) u\right) f\left(x+y+u e_{i}\right) d u d y
\end{aligned}
$$


Then for any $\bar{x} \in U$ the following inequalities

$$
\begin{aligned}
& \sup _{\bar{x} \in U}\left\|H_{\eta}^{i}\right\|_{q U_{\psi(\xi)}(\bar{x})} \leq C_{1}\left\|\left(\varphi_{i}(t)\right)^{-l_{i}} \Delta_{i}^{m_{i}}\left(\varphi_{i}(t), G_{\varphi(t)}\right) f\right\|_{p, \varphi, \beta ; G} \\
&\left.\times\left|Q_{\eta}^{i}\right| \prod_{j=1}^{n}\left(\psi_{j}([\xi]]_{1}\right)\right)^{\beta_{j} \frac{p}{q}} \\
& \sup _{\bar{x} \in U}\left\|H_{\eta T}^{i}\right\|_{q U_{\psi(\xi)}(\bar{x})} \leq C_{2}\left\|\left(\varphi_{i}(t)\right)^{-l_{i}} \Delta_{i}^{m_{i}}\left(\varphi_{i}(t), G_{\varphi(t)}\right) f\right\|_{p, \varphi, \beta ; G} \\
& \times\left|Q_{\eta T}^{i}\right| \prod_{j=1}^{n}\left(\psi_{j}\left([\xi]_{1}\right)\right)^{\beta_{j} \frac{p}{q}}, \\
& \sup _{\bar{x} \in U}\|A\|_{q U_{\psi(\xi)}(\bar{x})} \leq\|f\|_{p, \varphi, \beta ; G} \prod_{j=1}^{n}\left(\varphi_{j}(t)\right)^{-\nu_{j}-\left(1-\beta_{j} p\right)\left(\frac{1}{p}-\frac{1}{q}\right)} \prod_{j=1}^{n}\left(\psi_{j}[\xi]_{1}\right)^{\beta_{j} \frac{p}{q}}
\end{aligned}
$$

is hold, where $U_{\psi(\xi)}(\bar{x})=\left\{x:\left|x_{j}-\bar{x}_{j}\right|<\frac{1}{2} \psi_{j}(\xi), j=1,2, \ldots, n\right\}$ and $\psi \in N, C_{1}$, $C_{2}$ are the constants independent of $\varphi, \xi, \eta$ and $T$.

Proof. Applying sequentially the Minkowski generalized inequality for any $\bar{x} \in U$

$$
\left\|H_{\eta}^{i}\right\|_{q U_{\psi(\xi)}(\bar{x})} \leq \int_{0}^{\eta}\left\|L_{i}(\cdot, t)\right\|_{q U_{\psi(\xi)}(\bar{x})} \prod_{j=1}^{n}\left(\varphi_{j}(t)\right)^{\nu_{j}-2} \frac{\varphi_{i}^{\prime}(t)}{\varphi_{i}(t)} d t,
$$

and from the Hölder inequality $(q \leq r)$ we have

$$
\left\|L_{i}(\cdot, t)\right\|_{q U_{\psi(\xi)}(\bar{x})} \leq\left\|L_{i}(\cdot, t)\right\|_{r U_{\psi(\xi)}(\bar{x})} \prod_{j=1}^{n}\left(\psi_{j}(\xi)\right)^{\frac{1}{q}-\frac{1}{r}} .
$$

Now estimate the norm $\left\|L_{i}(\cdot, t)\right\|_{q U_{\psi(\xi)}(\bar{x})}$. Let $X$ be a characteristic function of the set $S\left(M_{i}\right)=$ supp $M_{i}$. Noting that $1 \leq p \leq r \leq \infty, s \leq r$, represent the integrand function (1.8) in the form

$$
\begin{aligned}
\left|\int_{-\infty}^{+\infty} M_{i} \zeta_{i} \Delta_{i}^{m_{i}} f d u\right| & =\left(\left|\int_{-\infty}^{+\infty} \zeta_{i} \Delta_{i}^{m_{i}} f d u\right|^{p}\left|M_{i}\right|^{s}\right)^{\frac{1}{r}} \\
& \times\left(\left|\int_{-\infty}^{+\infty} \zeta_{i} \Delta_{i}^{m_{i}} f d u\right|^{p} X\left(\frac{y}{\varphi(t)}\right)\right)^{\frac{1}{q}-\frac{1}{r}}\left(\left|M_{i}\right|^{s}\right)^{\frac{1}{s}-\frac{1}{r}}
\end{aligned}
$$

and apply to $\left|L_{i}\right|$ the Hölder inequality $\left(\frac{1}{p}+\left(\frac{1}{p}-\frac{1}{r}\right)+\left(\frac{1}{s}-\frac{1}{r}\right)=1\right)$, we obtain

$$
\begin{aligned}
\left\|L_{i}(\cdot, t)\right\|_{r, U_{\psi(\xi)}(\bar{x})} & \leq \sup _{x \in U_{\psi(\xi)}(\bar{x})}\left(\int_{R^{n}} \int_{-\infty}^{+\infty} \zeta_{i}\left(\frac{u}{\varphi_{i}(t)}, \frac{\rho_{i}\left(\varphi_{i}(t), x\right)}{\varphi_{i}(t)}, \frac{1}{2} \rho_{i}^{\prime}(\varphi(t), x)\right)\right. \\
& \left.\times\left.\Delta_{i}^{m_{i}}\left(\varphi_{i}(t)\right) f\left(x+y+u e_{i}\right) d u\right|^{p} \chi\left(\frac{y}{\varphi(t)}\right) d y\right)^{\frac{1}{p}-\frac{1}{r}} \\
& \times \sup _{y \in V}\left(\int_{U_{\psi(\xi)}(\bar{x})} \mid \int_{-\infty}^{+\infty} \zeta_{i}\left(\frac{u}{\varphi_{i}(t)}, \frac{\rho_{i}\left(\varphi_{i}(t), x\right)}{\varphi_{i}(t)}, \frac{1}{2} \rho_{i}^{\prime}(\varphi(t), x)\right)\right.
\end{aligned}
$$




$$
\begin{aligned}
& \left.\times\left.\Delta_{i}^{m_{i}}\left(\varphi_{i}(t) u\right) f\left(x+y+u e_{i}\right) d u\right|^{p} d x\right)^{\frac{1}{p}} \\
& \times\left(\int_{R^{n}}\left|M_{i}\left(\frac{y}{\varphi(t)}, \frac{\rho(\varphi(t), x)}{\varphi(t)}, \rho^{\prime}(\varphi(t), x)\right)\right|^{s} d y\right)^{\frac{1}{s}} .
\end{aligned}
$$

For any $x \in U$ we have

$$
\begin{aligned}
& \int_{R^{n}} \int_{-\infty}^{+\infty} \zeta_{i}\left(\frac{u}{\varphi_{i}(t)}, \frac{\rho_{i}\left(\varphi_{i}(t), x\right)}{\varphi_{i}(t)}, \frac{1}{2} \rho_{i}^{\prime}(\varphi(t), x)\right) \\
& \times\left.\Delta_{i}^{m_{i}}\left(\varphi_{i}(\delta) u\right) f\left(x+y+u e_{i}\right) d u\right|^{p} \chi\left(\frac{y}{\varphi(t)}\right) d y \\
& \leq \int_{(U+V)_{\varphi(t)}(\bar{x})}\left|\int_{-\infty}^{+\infty} \zeta_{i}\left(\frac{u}{\varphi_{i}(t)}, \frac{\rho_{i}\left(\varphi_{i}(t), x\right)}{\varphi_{i}(t)}, \frac{1}{2} \rho_{i}^{\prime}(\varphi(t), x)\right) \Delta_{i}^{m_{i}}\left(\varphi_{i}(\delta) u\right) f\left(y+u e_{i}\right) d u\right|^{p} \\
& \leq\left(\varphi_{i}(t)\right)^{p l_{i}} \| \int_{-\infty}^{+\infty} \zeta_{i}\left(\frac{u}{\varphi_{i}(t)}, \frac{\rho_{i}\left(\varphi_{i}(t), x\right)}{\varphi_{i}(t)}, \frac{1}{2} \rho_{i}^{\prime}(\varphi(t), x)\right) \varphi_{i}(t)^{-l_{i}} \\
& \times \Delta_{i}^{m_{i}}\left(\varphi_{i}(\delta) u, G_{\varphi(t)}\right) f d u \|_{p, G_{\varphi(t)}(x)}^{p} \\
& \leq \varphi_{i}(t)^{p+p l_{i}}\left\|\varphi_{i}(t)^{-l_{i}} \Delta_{i}^{m_{i}}\left(\varphi_{i}(\delta) u, G_{\varphi(t)}\right)\right\|_{p, \varphi, \beta}^{p} \prod_{j=1}^{n}\left(\varphi_{j}(t)\right)^{\beta_{j} p} .
\end{aligned}
$$

For $y \in V$ and $U_{\psi}+V \subset G_{\varphi}\left(\varphi\left([t]_{1}\right) \leq \psi\left([t]_{1}\right)\right)$

$$
\begin{gathered}
\int_{U_{\psi(\xi)}(\bar{x})}\left|\int_{-\infty}^{+\infty} \zeta_{i}\left(\frac{u}{\varphi_{i}(t)}, \frac{\rho_{i}\left(\varphi_{i}(t), x\right)}{\varphi_{i}(t)}, \frac{1}{2} \rho_{i}^{\prime}(\varphi(t), x)\right) \Delta_{i}^{m_{i}}\left(\varphi_{i}(\delta) u\right) f\left(x+y+u e_{i}\right) d u\right|^{p} d x \\
\leq \int_{G_{\varphi(\xi)}(\bar{x})}\left|\int_{-\infty}^{+\infty} \zeta_{i}\left(\frac{u}{\varphi_{i}(t)}, \frac{\rho_{i}\left(\varphi_{i}(t), x\right)}{\varphi_{i}(t)}, \frac{1}{2} \rho_{i}^{\prime}(\varphi(t), x)\right) \Delta_{i}^{m_{i}}\left(\varphi_{i}(\delta) u\right) f\left(x+u e_{i}\right) d u\right|^{p} d x \\
\leq\left(\varphi_{i}(t)\right)^{p l_{i}} \mid \int_{-\infty}^{+\infty} \zeta_{i}\left(\frac{u}{\varphi_{i}(t)}, \frac{\rho_{i}\left(\varphi_{i}(t), x\right)}{\varphi_{i}(t)}, \frac{1}{2} \rho_{i}^{\prime}(\varphi(t), x)\right) \\
\times \varphi_{i}(t)^{-l_{i}} \Delta_{i}^{m_{i}}\left(\varphi_{i}(\delta) u, G_{\varphi(t)}\right) f d u \|_{p, G_{\varphi(t)}(\bar{x})}^{p} \\
\quad \leq \varphi_{i}(t)^{p+p l_{i}}\left\|\varphi_{i}(t)^{-l_{i}} \Delta_{i}^{m_{i}}\left(\varphi_{i}(\delta), G_{\varphi(t)}\right)\right\|_{p, \varphi, \beta}^{p} \prod_{j=1}^{n}\left(\psi_{j}\left([\xi]_{1}\right)\right)^{\beta_{j} p} \\
\quad \int_{R^{n}}\left|M_{i}\left(\frac{y}{\varphi(t)}, \frac{\rho(\varphi(t), x)}{\varphi(t)}, \rho^{\prime}(\varphi(t), x)\right)\right|^{s} d y=\left\|M_{i}\right\|_{s}^{s} \cdot \prod_{j=1} \varphi_{j}(t) .
\end{gathered}
$$

From inequalities (1.14)-(1.17) it follows that

$$
\begin{aligned}
\left\|L_{i}(\cdot, t)\right\|_{r U_{\psi(\xi)}(\bar{x})} & \leq\left\|M_{i}\right\|_{s} \cdot\left\|\left(\varphi_{i}(t)\right)^{-l_{i}} \Delta_{i}^{m_{i}}\left(\varphi_{i}(\delta)\right) f\right\|_{p, \varphi, \beta ; G}\left(\varphi_{i}(t)\right)^{1+l_{i}} \\
& \times \prod_{j=1}^{n}\left(\varphi_{j}(t)\right)^{\frac{1}{s}+\beta_{j} p\left(\frac{1}{p}-\frac{1}{r}\right)} \cdot \prod_{j=1}^{n}\left(\psi_{j}\left([\xi]_{1}\right)\right)^{\frac{\beta_{j} p}{r}} .
\end{aligned}
$$


Inequality (1.11) is proved analogously.

Inequalities (1.12), (1.13) and (1.18) for $r=q$ and for any $\bar{x} \in U$ reduce to the estimation

$$
\begin{aligned}
\left\|H_{\eta}^{i}\right\|_{r U_{\psi(\xi)}(\bar{x})} & \leq C_{1}\left\|\left(\varphi_{i}(t)\right)^{-l_{i}} \Delta_{i}^{m_{i}}\left(\varphi_{i}(\delta)\right) f\right\|_{p, \varphi, \beta ; G} \\
& \times\left|Q_{\eta}^{i}\right| \prod_{j=1}^{n}\left(\psi_{j}\left([\xi]_{1}\right)\right)^{\beta_{j} \frac{p}{q}} \quad\left(Q_{\eta}^{i}<\infty\right) .
\end{aligned}
$$

In the case $Q_{\eta, T}^{i}<\infty$ inequality (1.10) can be proved in the same way.

From inequality (1.18) for $r=q$ and (1.19) we get the inequality $(\forall \bar{x} \in U)$

$$
\begin{aligned}
& \sup _{\bar{x} \in U}\left\|L_{i}\right\|_{q U_{\psi(\xi)}(\bar{x})} \leq C_{2}\left\|\left(\varphi_{i}(t)\right)^{-l_{i}} \Delta_{i}^{m_{i}}\left(\varphi_{i}(t), G_{\varphi(t)}\right) f\right\|_{p, \varphi, \beta ; G} \cdot \prod_{j=1}^{n}\left(\psi_{j}\left([\xi]_{1}\right)\right)^{\beta_{j} \frac{p}{q}} \\
& \sup _{\bar{x} \in U}\left\|H_{\eta}^{i}\right\|_{q U_{\psi(\xi)}(\bar{x})} \leq C_{3}\left\|\left(\varphi_{i}(t)\right)^{-l_{i}} \Delta_{i}^{m_{i}}\left(\varphi_{i}(t), G_{\varphi(t)}\right) f\right\|_{p, \varphi, \beta ; G} \cdot \prod_{j=1}^{n}\left(\psi_{j}\left([\xi]_{1}\right)\right)^{\beta_{j} \frac{p}{q}} .
\end{aligned}
$$

From last inequalities it follows that

$$
\begin{gathered}
\left\|L_{i}\right\|_{q, \psi, \beta^{1} ; U} \leq C_{1}^{\prime}\left\|\left(\varphi_{i}(t)\right)^{-l_{i}} \Delta_{i}^{m_{i}}\left(\varphi_{i}(t), G_{\varphi(t)}\right) f\right\|_{p, \varphi, \beta ; G}, \\
\left\|H_{\eta}^{i}\right\|_{q, \psi, \beta^{1} ; U} \leq C_{2}^{\prime}\left\|\left(\varphi_{i}(t)\right)^{-l_{i}} \Delta_{i}^{m_{i}}\left(\varphi_{i}(t), G_{\varphi(t)}\right) f\right\|_{p, \varphi, \beta ; G} .
\end{gathered}
$$

$C_{1}^{\prime}$ and $C_{2}^{\prime}$ are the constants independent of $\varphi$.

\section{Main Results}

Prove two theorems on the properties of the functions from the space $H_{p, \varphi, \beta}^{l}(G, \lambda)$.

Theorem 2.1. Let $G \subset R^{n}$ satisfy the condition of flexible $\varphi$-horn, $1 \leq p \leq q \leq \infty, \nu=\left(\nu_{1}, \nu_{2}, . ., \nu_{n}\right)$, $\nu_{j} \geq 0$ be entire $j=1,2, \ldots, n, Q_{T}^{i}<\infty(i=1,2, \ldots, n)$ and let $f \in \bar{H}_{p, \varphi, \beta}^{l}(G, \lambda)$. Then the following embeddings hold

$$
D^{\nu}: H_{p, \varphi, \beta}^{l}(G) \rightarrow L_{q, \psi, \beta^{1}}(G)
$$

more precisely, for $f \in H_{p, \varphi, \beta}^{l}(G, \lambda)$ there exists a generalized derivative $D^{\nu} f$ and the following inequalities are valid

$$
\begin{aligned}
&\left\|D^{\nu} f\right\|_{q, G} \leq C_{1}\left(B(t)\|f\|_{q, \psi, \beta ; G}\right. \\
&\left.+\sum_{i=1}^{n}\left|Q_{T}^{i}\right| \sup _{0<t<t_{0}}\left\|\frac{\Delta_{i}^{m_{i}}\left(\varphi_{i}(t), G_{\varphi(t)}\right) f}{\left(\varphi_{i}(t)\right)^{l_{i}}}\right\|_{p, \varphi, \beta ; G}\right), \\
&\left\|D^{\nu} f\right\|_{q, \psi, \beta^{1} ; G} \leq C_{2}\|f\|_{H_{p, \varphi, \beta}^{l}(G, \lambda)}, p \leq q<\infty .
\end{aligned}
$$

In particular, if

$$
Q_{T, 0}^{i}=\int_{0}^{T} \prod_{j=1}^{n}\left(\varphi_{j}(t)\right)^{-\nu_{j}-\left(1-\beta_{j} p\right) \frac{1}{p}} \frac{\varphi_{i}^{\prime}(t)}{\left(\varphi_{i}(t)\right)^{1-l_{i}}} d t<\infty,(i=1,2, \ldots, n),
$$


then $D^{\nu} f(x)$ is continuous on $G$, i.e

$$
\begin{aligned}
\sup _{x \in G}\left|D^{\nu} f(x)\right| \leq & C_{1}\left(B(t)\|f\|_{p, \varphi, \beta ; G}\right. \\
& \left.+\sum_{i=1}^{n}\left|Q_{T, 0}^{i}\right| \sup _{0<t<t_{0}} \|\left.\frac{\Delta_{i}^{m_{i}}\left(\varphi_{i}(t), G_{\varphi(t)}\right) f}{\left(\varphi_{i}(t)\right)^{l_{i}}}\right|_{p, \varphi, \beta ; G}\right)
\end{aligned}
$$

$0<T \leq \min \left\{1, T_{0}\right\}, T_{0}$ is a fixed number; $C_{1}, C_{2}, C_{3}, C_{4}$ are the constants independent of $f$, also $C_{1}$ and $C_{3}$ are independent from $T$.

Proof. At first note that in the conditions of our theorem there exists a generalized derivative $D^{\nu} f$ on $G$. Indeed, from the condition $Q_{T}^{i}<\infty$ for all $(i=1,2, \ldots, n)$ it follows that for $f \in H_{p, \varphi, \beta}^{l}(G) \rightarrow H_{p}^{l}(G)$, there exists $D^{\nu} f \in L_{p}(G)$ and for integral representation (1.3) and (1.4) with the same kernels is valid.

Applying the Minkowski inequality, from identities (1.3) and (1.4) we get

$$
\left\|D^{\nu} f\right\|_{q, G} \leq\left\|f_{\varphi(T)}^{(\nu)}\right\|_{q, G}+\sum_{i=1}^{n}\left\|H_{T}^{i}\right\|_{q, G}
$$

By means of inequality (1.11) for $U=G, M_{i}=K_{i}^{i}, t=T$ we get

$$
\begin{aligned}
\left\|f_{\varphi(t)}^{(\nu)}\right\|_{q, G} & \leq\|f\|_{p, \varphi, \beta ; G} \prod_{j=1}^{n}\left(\varphi_{j}(t)\right)^{-\nu_{j}-\left(1-\beta_{j} p\right)\left(\frac{1}{p}-\frac{1}{q}\right)} \prod_{j=1}^{n}\left(\psi_{j}\left([\xi]_{1}\right)\right)^{\beta_{j} \frac{p}{q}} \\
& \leq C_{1} A(t)\|f\|_{p, \varphi, \beta ; G}
\end{aligned}
$$

and by means of inequality (1.9) for $\eta=T, M_{i}=K_{i}^{i}, U=G$, we get

$$
\left\|H_{T}^{i}\right\|_{q, G} \leq C_{2} Q_{T}^{i}\left\|\left(\varphi_{i}(t)\right)^{-l_{i}} \Delta_{i}^{m_{i}}\left(\varphi_{i}(t), G_{\varphi(t)}\right) f\right\|_{p, \varphi, \beta ; G} .
$$

Substituting (2.7) and (2.6) in (2.5), we get inequality (1.21). By means of inequalities (1.20) and (1.21) for $\eta=T$ we get inequality $(2.2)$.

Now let conditions (2.3) be satisfied, then take into account identities (1.3), (1.4), from inequality (2.5) we get

$$
\left\|D^{\nu} f-f_{\varphi(T)}^{(\nu)}\right\|_{\infty, G} \leq C \sum_{i=1}^{n}\left|Q_{T}^{i}\right| \sup _{0<t<t_{0}}\left\|\frac{\Delta_{i}^{m_{i}}\left(\varphi_{i}(t), G_{\varphi(t)}\right) f}{\left(\varphi_{i}(t)\right)^{l_{i}}}\right\|_{p, \varphi, \beta ; G} .
$$

As $T \rightarrow 0$, the left side of this inequality tends to zero, since $f_{\varphi(T)}^{(\nu)}(x)$ is continuous on $G$ and the convergence on $L_{\infty}(G)$ coincides with the uniform convergence. Then the limit function $D^{\nu} f$ is continuous on $G$.

Let $\gamma$ be an $n$-dimensional vector.

Theorem 2.2. Let all the conditions of Theorem 1 be fulfilled. Then for $Q_{T}^{i}<\infty(i=1,2, \ldots, n)$ the derivative $D^{\nu} f$ satisfies on $G$ the Hölder generalized condition, i.e the following inequality is valid:

$$
\left\|\Delta(\gamma, G) D^{\nu} f\right\|_{q, G} \leq C\|f\|_{H_{p, \varphi, \beta}^{l}(G)} \cdot|h(|\gamma|, \varphi ; T)|,
$$

where $C$ is a constant independent of $f,|\gamma|$ and $T$.

In particular, if $Q_{T, 0}^{i}<\infty,(i=1,2, \ldots, n)$, then

$$
\sup _{x \in G}\left|\Delta(\gamma, G) D^{\nu} f(x)\right| \leq C\|f\|_{H_{p, \varphi, \beta}^{l}\left(G_{\varphi}\right)} \cdot\left|h_{0}(|\gamma|, \varphi, T)\right| .
$$

where $h(|\gamma|, \varphi, T)=\max _{i}\left\{|\gamma|, Q_{|\gamma|}^{i}, Q_{|\gamma|, T}^{i}\right\}\left(h_{0}(|\gamma|, \varphi, T)=\max _{i}\left\{|\gamma|, Q_{|\gamma|, 0}^{i}, Q_{|\gamma|, T, 0}^{i}\right\}\right)$ 
Proof. According to Lemma 8.6 from [1] there exists a domain

$$
G_{\omega} \subset G(\omega=\zeta r(x), \zeta>0 r(x)=\rho(x, \partial G), x \in G)
$$

and assume that $|\gamma|<\omega$, then for any $x \in G_{\omega}$ the segment connecting the points $x, x+\gamma$ is contained in $G$. Consequently, for all the points of this segment, identities (1.3), (1.4) with the same kernels are valid. After the same transformations, from (1.3) and (1.4) we get

$$
\begin{aligned}
& \left|\Delta(\gamma, G) D^{\nu} f(x)\right| \leq \prod_{j=1}^{n}\left(\varphi_{j}(t)\right)^{-1-\nu_{j}} \\
& \times \int_{R^{n}} \int_{R^{n}}|f(x+y+z)| \mid \Omega^{(\nu)}\left(\frac{y-\gamma}{\varphi(t)}, \frac{\rho(\varphi(t), x)}{2 \varphi(t)}\right) \\
& -\Omega^{(\nu)}\left(\frac{y}{\varphi(t)}, \frac{\rho(\varphi(t), x)}{2 \varphi(T)}\right) \mid d y d z \\
& +C_{2} \sum_{i=1}^{n}\left\{\int_{0}^{|\gamma|} \int_{R^{n}}^{+\infty}\left|\zeta_{i}\left(\frac{u}{\varphi_{i}(t)}, \frac{\rho_{i}\left(\varphi_{i}(t, x)\right)}{\varphi_{i}(t)}, \frac{1}{2} \rho^{\prime}(\varphi(t), x)\right)\right| \times-\right. \\
& \times\left|K_{i}^{(\nu)}\left(\frac{y}{\varphi(t)}, \frac{\rho(\varphi(t, x))}{\varphi(t)}\right)\right|\left|\Delta_{i}^{m_{i}}\left(\varphi_{i}(\delta) u\right) f\left(x+y+u e_{i}\right)\right| d y d u d t \\
& +\int_{|\gamma|}^{T} \int_{R^{n}}^{+\infty}\left|K_{i}^{(\nu)}\left(\frac{y}{\varphi(t)}, \frac{\rho(\varphi(t, x))}{\varphi(t)}\right)\right|\left|\zeta_{i}\left(\frac{u}{\varphi_{i}(t)}, \frac{\rho_{i}\left(\varphi_{i}(t, x)\right)}{\varphi_{i}(t)}, \frac{1}{2} \rho_{i}^{\prime}(\varphi(t), x)\right)\right| \\
& \left.\quad \int_{1}\left|\Delta_{i}^{m_{i}}\left(\varphi_{i}(\delta) u\right) f(x+y+v \gamma)\right| d v d u d y d t\right\} . \\
& =C_{1} A(x, \gamma)+C_{2} \sum_{i=1}^{n}(E(x, \gamma)+F(x, \gamma)),
\end{aligned}
$$

where $0<T \leq\left\{1, T_{0}\right\}$. Additionally, we assume that $|\gamma|<T$. Consequently, $|\gamma|<\min (\omega, T)$. If $x \in G \backslash G_{\omega}$, then

$$
\Delta(\gamma, G) D^{\nu} f(x)=0 .
$$

By inequality (2.9) we have

$$
\begin{gathered}
\left\|\Delta(\gamma, G) D^{\nu} f\right\|_{q, G} \leq\|A(\cdot, \gamma)\|_{q, G_{\omega}} \\
+\sum_{i=1}^{n}\left(\|E(\cdot, \gamma)\|_{q, G_{\omega}}+\|F(\cdot, \gamma)\|_{q, G_{\omega}}\right), \\
A(x, \gamma) \leq \prod_{j=1}^{n}\left(\varphi_{j}(t)\right)^{-\nu_{j}-2} \int_{0}^{|\gamma|} d \zeta \int_{R^{n}} \int_{R^{n}}\left|f\left(x+\zeta e_{\gamma}+y\right)\right| \\
\times\left|D_{j} \Omega^{(\nu)}\left(\frac{y}{\varphi(T)}, \frac{\rho(\varphi(t), x)}{2 \varphi(t)}\right) \Omega^{(\nu)}\left(\frac{z}{\varphi(T)}, \frac{\rho(\varphi(t), x)}{2 \varphi(t)}\right)\right| d y d z .
\end{gathered}
$$

Taking into account $\xi e_{\gamma}+G_{\omega} \subset G$, and applying the generalized Minkowski inequality, from inequality (1.11) for $U=G$, we have

$$
\|A(\cdot, \gamma)\|_{q, G_{\omega}} \leq C_{1}|\gamma|\|f\|_{p, \varphi, \beta ; G} .
$$

By means of inequality (1.9), for $U=G, \eta=|\gamma|$ we get

$$
\|E(\cdot, \gamma)\|_{q, G_{\omega}} \leq C_{2}\left|Q_{|\gamma|}^{i}\right|\left\|\left(\varphi_{i}(t)\right)^{-l_{i}} \Delta_{i}^{m_{i}}\left(\varphi_{i}(t), G_{\varphi(t)}\right) f\right\|_{p, \varphi, \beta ; G}
$$


and by means of inequality (1.10) for $U=G, \eta=|\gamma|$ we get

$$
\|F(\cdot, \gamma)\|_{q, G_{\omega}} \leq C_{3}\left|Q_{|\gamma|, T}^{i}\right|\left\|\left(\varphi_{i}(t)\right)^{-l_{i}} \Delta_{i}^{m_{i}}\left(\varphi_{i}(t), G_{\varphi(t)}\right) f\right\|_{p, \varphi, \beta ; G} .
$$

From inequalities (2.11) and (2.12)-(2.14) we get the required inequality.

Now suppose that $|\gamma| \geq \min (\omega, T)$. Then

$$
\left\|\Delta(\gamma, G) D^{\nu} f\right\|_{q, G} \leq 2\left\|D^{\nu} f\right\|_{q, G} \leq C(\omega T)\left\|D^{\nu} f\right\|_{q, G}|h(|\gamma|, \varphi ; T)| .
$$

Estimating for $\left\|D^{\nu} f\right\|_{q, G}$ by means of inequality (2.1), in this case we get estimation (2.8).

Acknowledgments. The research of A. Najafov was partially supported by the grant of Presidium Azerbaijan National Academy of Science 2015.

\section{References}

1. O.V. Besov, V.P. Il'yin. S. M. Nikolskii, Integral representations of functions and embeddings theorems, M. Nauka, 1996, 480 p.

2. A. Gogatishvili, R. Ch. Mustafayev, On a theorem of Muchenhoupt-Wheeden in generalized Morrey spaces, Eurasian Mathematical Journal, 2 (2) 2011, 134-138.

3. V.S. Guliyev and R.Ch. Mustafayev, Boundedness of the anisotropic maximal and anisotropic singular integral operators in generalized Morrey spaces, Acta Mathematica Sinica-English series, 27 (12), 2011, 2361-2370.

4. V.S. Guliyev, Y. Sawano. Linear and sublinear operators on Generalized Morrey spaces with non-doubling measures, Publicationes Mathematicae Debrecen, vol. 83, 2013, no. 3, 1-17.

5. V.P. Il'yin. On some properties of the functions of spaces $W_{p, a, \chi}^{l}(G)$, Zap. Nauch.Sem. LOMI AN SSSR, v.2, 1971, 33-40.

6. L. Lanzhe, Interior estimates in Morrey spaces for solutions of elliptic equations and weighted boundedness for commutators of singular integral operators, 25 B(1), 2005, 89-94.

7. C.B. Morrey, On the solutions of quasi-linear elliptic partial differential equations, Trans. Amer. Math. Soc., 43(1938), 126-166.

8. E. Nakai, Hardy-Littlewood maximal, singular integral operator and Riesz potentials on generalized Morrey spaces, Math. Nach., 166, 1994, 95-103.

9. A.M. Najafov, On some properties of the functions from Sobolev-Morrey type spaces, Central European Journal of Math., 3(3), 2005, 496-507.

10. A.M. Najafov, On some properties of functions the Sobolev-Morrey type spaces $W_{p, a, \chi, \tau}^{l}(G)$, Siberian Math. Journal, 46(3), 2005, 634-648.

11. A.M. Najafov, Some properties of functions from the intersection of Besov-Morrey type spaces with dominant mixed derivatives, Proc. of A. Razmadze Math. Inst., v. 139, 2005, 71-82.

12. A.M. Najafov, The embedding theorems of spaces $W_{p, \varphi, \beta}^{l}(G)$, Mathematica Aeterna, 3(4), 2013, 299-308.

13. J. Ross, A Morrey-Nikolskii inequality, Proc. Amer. Math. Soc. 78, 1980, 97-102. 